\title{
Aplicação Móvel Para Avaliação Postural Usando Visão Computacional
}

\author{
Helano M. B. F. Portela* Luis F. M. S. Silva** \\ Rodrigo M. S. Veras ${ }^{* * *}$ Vinicius P. Machado**** \\ * Universidade Federal Do Piauí, (e-mail: helano38@gmail.com). \\ ** Instituo Federal Do Maranhão, (e-mail: luis.maia@ifma.edu.br) \\ *** Universidade Federal Do Piauí, (e-mail: rveras@ufpi.edu.br) \\ **** Universidade Federal Do Piauí, (e-mail: vinicius@ufpi.edu.br )
}

\begin{abstract}
Usually, the previous diagnosis of postural deviations is made by visual methods or using images. This approach uses qualitative parameters, which are susceptible to interference by the examiner and may lead to changes in the results. Performing an accurate diagnosis allied with a treatment follow-up requires a large number of equipments. Therefore, it is relevant to improve and to cheapen such process using more accessible devices. This work presents the development of a mobile application that uses image processing and fotogrammetry concepts capable of helping in the diagnosis of a postural deviation known as scoliosis. The goal is to enforce the hypothesis that the use of image processing is capable of measuring angles related to scoliosis in human beings using images provided by smartphones.

Resumo: O diagnóstico prévio de desvios posturais é feito, na maioria das vezes, de forma visual ou com o auxílio de imagens. Em ambos os casos, faz-se uso de parâmetros qualitativos, que por sua vez são suscetíveis à interferência do examinador e podem culminar em alterações no resultado. A obtenção de um diagnóstico preciso, aliado com o acompanhamento do tratamento, requer uma grande quantidade de equipamentos. Portanto, torna-se um problema relevante encontrar meios de aperfeiçoar e baratear esse processo usando dispositivos mais acessíveis. Dessa forma, o presente trabalho apresenta o desenvolvimento de uma aplicação para smartphones capaz de auxiliar na detecção de medidas fotogramétricas de desvios posturais caracterizados como escoliose. A abordagem desenvolvida visa testar a hipótese de que o uso de processamento e análise de imagens é capaz de identificar ângulos relacionados a escoliose em humanos usando imagens provenientes de smartphones.
\end{abstract}

Keywords: Posture Measurement; Mobile Applications; Image processing; Postural Deviations; Scoliosis; Computer Vision.

Palavras-chaves: Avaliação Postural; Aplicações Móveis; Processamento de Imagens; Desvios Posturais; Escoliose; Visão Computacional.

\section{INTRODUÇÃO}

A escoliose caracteriza-se como uma deformação da coluna em três planos no espaço, no qual uma perturbação causa um movimento de torção generalizado em toda a coluna, originando uma quebra no equilíbrio raquidiano. A escoliose pode se desenvolver como uma curva primária em formato de (C) ou com duas curvas, uma curva primária seguida de uma curva de compensação, caracterizadas em formato de $(\mathrm{S})$. Esta condição pode se desenvolver na área torácica ou na área lombar, sendo mais frequente entre essas duas áreas (lombar e torácica). O nível de gravidade da escoliose é determinado pela extensão da curvatura e pelo ângulo de rotação do tronco (Suken A. Shah, 2009).

O método mais apropriado para avaliação de curvas relacionadas a escoliose é a partir dos ângulos de Cobb diretamente sobre radiografias (Suken A. Shah, 2009). Entretanto, além de expor o indivíduo aos efeitos da radiação, envolve um custo significativo e nem sempre esse tipo de exame está disponível ao avaliador (Stolfi, 2014). Desse modo torna-se mais viável, para o estudo de desvios posturais, a avaliação postural através de testes não invasivos.

Nos últimos anos, alguns trabalhos vêm utilizando técnicas de fotogrametria em testes com imagem (Nery, 2009; Stolfi, 2014). A fotogrametria possibilita a verificação da simetria de segmentos corporais (Nery, 2009) com base em pontos anatômicos demarcados no corpo do indivíduo, sendo possível analisar a verticalização e horizontalização do corpo humano (Stolfi, 2014).

Sendo um problema relevante encontrar meios de aperfeiçoar e baratear o processo de avaliação postural usando dispositivos mais acessíveis, a fim de executar avaliações posturais através de testes não invasivos. Surge a possibilidade do uso da técnica de fotogrametria aliada ao processamento de imagens, o que leva a seguinte questão de pesquisa: é possível identificar escolioses usando processamento de imagens e fotogrametria em smartphones? Assim, a pesquisa teve como objetivo geral desenvolver uma aplicação para smartphones capaz de auxiliar na detecção 
da escoliose usando processamento de imagem, de forma a proporcionar ao profissional de saúde um diagnóstico e acompanhamento otimizado.

Com vistas a alcançar o objetivo geral foram traçados três objetivos específicos. O primeiro objetivo é o desenvolvimento de um algoritmo capaz de: identificar através de imagens os pontos fotogramétricos necessários para a análise da escoliose; Traçar segmentos de retas a partir dos pontos fotogramétricos identificados; Calcular o ângulo formado pelos seguimentos de reta traçados entre os pontos fotogramétricos. O segundo objetivo é a construção de um aplicativo móvel para avaliação postural a partir desse algoritmo. E por fim, o último objetivo especifico é a validação da abordagem proposta.

Alguns trabalhos já foram desenvolvidos com o intuito de realizar diagnóstico de desvios posturais. Por exemplo, a pesquisa de Srinivasalu et al. (2008) teve como objetivo analisar a confiabilidade inter e intra-examinador da avaliação digital de radiografias. Na metodologia aplicada na pesquisa, três avaliadores executaram o aferimento de 318 radiografias de crianças em estado pre-operatório diagnosticadas com escoliose idiopática. Apenas uma curva foi aferida por radiografia. Cada avaliador aferiu o ângulo de Cobb três vezes em um intervalo de uma semana entre as avaliações. O sistema computacional em questão (PACS, PiViewSTAR version 5) foi usado para aferição em todos os casos. A vértebra final foi pré-selecionada para evitar uma potencial fonte de erro na medição. Os resultados da pesquisa levaram a conclusão que o método de avaliação digital através do software minimiza a discrepância entre as avaliações (Srinivasalu et al., 2008).

O trabalho de Stolfi (2014) teve como objetivo verificar a confiabilidade intra e inter-examinador de medidas fotogramétricas na verificação do alinhamento vertical da coluna vertebral. O estudo foi realizado com 30 individuos, cujos pontos anatômicos foram demarcados com base na técnica de anatomia palpatória e posteriormente foram feitos registros fotográficos de cada participante. A análise fotogramétrica foi feita no software $S A P O$, usando as ferramentas de ângulos livres e ângulo com a vertical (processo executado por dois examinadores experientes).

Com base nos dados resultantes da pesquisa, Stolfi (2014) concluíram que a técnica aplicada se mostrou confiável nos dois quesitos analisados, tanto intra quanto interexaminador. Semelhantemente ao relatado por Souza et al. (2011), que utilizaram uma abordagem mais completa, efetuando avaliações nas vistas anterior, posterior e sagital e, além disso, considerando uma quantidade maior de pontos anatômicos.

O presente trabalho se difere da abordagem desenvolvida por Stolfi (2014) e Souza et al. (2011) ao propor uma ferramenta capaz de identificar as marcações fotogramétricas automaticamente e calcular os ângulos necessários para a análise vertical da coluna vertebral, além de propor o registro e análise a partir de um aplicativo que executa em dispositivos móveis.

O trabalho de Shaw et al. (2012) propôs uso do acelerômetro existente em smartphones para aferir o ângulo de Cobb e compará-lo ao método tradicional em testes com 20 pacientes que apresentavam escoliose idiopática.
Na metodologia utilizada, sete observadores aferiram os ângulos de Cobb a partir da radiografia de 20 pacientes em pré-operatório com ambos os métodos, tradicional e com o smartphones. Com base nos resultados obtidos na pesquisa, o trabalho concluiu que o método proposto obteve resultados equivalentes ao método tradicional, mas com a vantagem de diminuir em $15 \%$ o tempo necessário para executar o mesmo processo (Shaw et al., 2012).

O trabalho de Mazzuia et al. (2015) apresenta os resultados de um experimento que compara a aferição do ângulo de Cobb a partir de duas abordagens diferentes. A primeira é descrita como a abordagem tradicional, e é realizada delimitando-se em anteroposterior as vértebras mais cranial e mais caudal em determinada curva nas radiografias da coluna total. Tradicionalmente, essa medida manual é feita com lápis e goniômetro. O segundo método, chamado CobbMeter, é capaz de aferir o ângulo de Cobb nas radiografias por meio do sensor de angulação disponível no Iphone.

Na prática, $2,08^{\circ}$ de diferença para mais ou menos $\left(4,16^{\circ} \mathrm{em}\right.$ absoluto) na medição de uma curva de escoliose não traduz significado preocupante que venha causar erros interpretativos para diagnóstico, estabelecimento de prognóstico ou definição do tratamento. Esses desvios são obtidos mesmo intra ou inter-examinador na aferição manual rotineira. Desse modo, o uso do aplicativo CobbMeter para aferição de ângulos de radiografias em estudo de escoliose idiopática do adolescente pode ser considerado seguro e aplicável na prática clínica (Mazzuia et al., 2015).

O objetivo do trabalho de Iacob et al. (2018) foi determinar se o aplicativo móvel Posture Screen Mobileß) é uma ferramenta útil para para a avaliação da correlação entre má oclusão e desvios posturais corporal. O estudo envolveu 29 participantes que passaram por exame de oclusão dentaria, tanto estática quanto dinâmica e por avaliação postural com uso do aplicativo Posture Screen Mobileß). O participantes foram divididos em grupos de acordo com as características de oclusão dentaria que apresentavam, os parâmetros provenientes das avaliações posturais foram então comparados entre esses grupos.

Segundo os resultados obtidos, foi evidenciada a correlação entre desvios posturais e a oclusão dentária. A pesquisa concluiu que o aplicativo móvel usado para a avaliação postural se caracterizou como uma ferramenta útil e acessível para o problema proposto.

Por fim, o trabalho de Szucs and Brown (2018) realizou um estudo para determinar a confiabilidade e a validade do uso de uma aplicação móvel para avaliação postural em jovens adultos. Na realização do estudo foi utilizado o aplicativo Posture Screen Mobile $\mathbb{R}$ para realizar a análise de 20 indivíduos. Como conclusão o trabalho indica que a aplicação demostrou uma forte confiabilidade, além de outros pontos positivos como : custo beneficio; possibilidade de análise de uma maior quantidade de variáveis em relação às ferramentas clínicas; Fácil interface de uso.

\subsection{Ferramentas Disponíveis}

Software SAPO: O projeto $S A P O$ visa disponibilizar uma ferramenta desktop de código aberto para procedimentos científicos de avaliação postural. O software possui 
uma documentação que indica um protocolo de marcação de pontos posturais para serem delimitados na realização da avaliação postural. Dessa forma, o avaliador possui uma lista de pontos que deverão ser demarcados no corpo do paciente para vista anterior, posterior e lateral. Através da ferramenta de ângulos livres, o avaliador tem a possibilidade de interligar os pontos posturais, gerando assim medidas de ângulos que serão interpretados para aferir a condição postural do paciente (Duarte M., 2005). O presente trabalho, se diferencia ao propor uma ferramenta de avaliação postural voltada para dispositivos móveis, e além disso, propor o uso da visão computacional para a identificação automática dos pontos demarcados no paciente.

Posture Screen Mobile(R): Aplicação é voltada para profissionais da saúde como quiropraticistas, educadores físicos e massoterapeutas, que necessitam avaliar a postura de seus pacientes. Nessa aplicação, a avaliação é feita a partir da captura de uma imagem do paciente. Depois dessa etapa, o avaliador tem a possibilidade de marcar manualmente na imagem, através da tela do celular, os pontos base do corpo do paciente, que serão usados na avaliação. Um ponto importante a se destacar é que a confiabilidade da escolha dos pontos fotogramétricos é baixa, pois, segundo a literatura referente a avaliação postural, o ideal é detectar os pontos fotogramétricos a partir de técnicas de anatomia palpatória seguindo um protocolo específico, o que não ocorre neste software.

O trabalho de Hopkins et al. (2019) tem o objetivo de validar e verificar a confiabilidade da ferramenta Posture Screen Mobileß . A partir dos resultados o autor comenta que o uso de marcadores anatômicos na tela não melhorou o processo de mensuração, além disso indica que a ferramenta não seja usada quando a avaliação precisar obter dados quantitativos precisos.

\section{MÉTODO PROPOSTO}

Para a execução da análise, o algoritmo proposto segue cinco etapas (Figura 1): conversão da imagem; segmentação; definição de Contornos; identificação dos pontos; e cálculo dos angulos.

A seguir são detalhadas as etapas do diagrama acima, que buscam realizar a identificação dos pontos fotogramétricos e cálculo dos ângulos.

\subsection{Conversão da Imagem}

Nesta etapa temos que considerar uma questão importante: Qual característica da imagem indica as marcações que devem ser identificadas? Se as marcações são de cor branca ou reflexivas, é possível melhorar o desempenho nas etapas posteriores do processamento convertendo a imagem original de RGB para escala de cinza, que possui apenas um canal.

Caso as marcações sejam de cores específicas, a imagem então é convertida para o formato HSV, que é um sistema de cores mais adequado para aplicações que objetivam realizar segmentação com base em características de coloração. Por exemplo, se a marcação for de cor verde, o algoritmo deve realizar segmentação das áreas candidatas representadas no espectro verde.

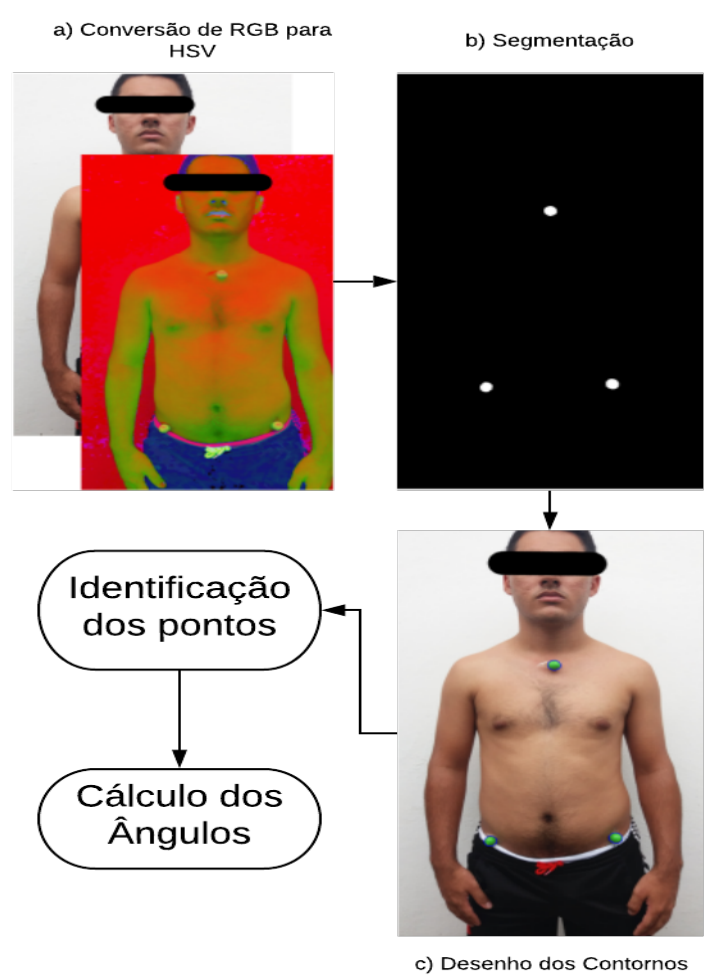

Figura 1. Diagrama sequencial de etapas realizadas pelo algoritmo.

\subsection{Segmentação}

Uma das formas de se realizar segmentação envolve um método chamado Thresholding, que é capaz de enfatizar ou separar as partes da imagem que se deseja analisar. Neste caso, o valor de cada pixel é comparado com um valor de segmentação predefinido, e com base no resultado da comparação o valor do pixel é modificado. Para a construção da aplicação é necessário que o algoritmo seja capaz de segmentar áreas da imagem contendo características que indiquem a existência de marcações de pontos fotogramétricos (Kapur and Thakkar, 2015).

Para esse trabalho, o valor predefinido de Thresholding para identificação de marcações brancas (em imagens em escala de cinza) foi 200 , visto que o valor máximo de representação do pixel é 255, e o valor mínimo 0. Logo, são segmentadas regiões de brilho mais intenso. Para efetuar essa operação é usado o método threshold da classe Imgproc (OpenCV). Os parâmetros de entrada para o método são: Imagem em escala de cinza; Objeto Mat que receberá a matriz de pixels como o resultado da operação; valor inteiro do Threshold; valor máximo e, por fim, a flag THRESH_BINARY, representando o tipo de Threshold a ser utilizado.

No caso de marcações verdes, a segmentação ocorre com base nas características de cor. Portanto foi definida uma faixa de valores do sistema de cores HSV, para matiz, saturação e intensidade. Foram definidos os respectivos valores compreendidos entre $(30,85,40)$ como limite mínimo e $(75,255,255)$ como limite máximo. As áreas de uma imagem que satisfaçam as características contidas nessa faixa de cores são obtidas pelo método inRange disponível pela $O p e n C V$. 


\subsection{Desenho dos Contornos}

Após a segmentação da imagem, precisamos identificar os pontos fotogramétricos encontrando sua forma e posição, para podermos então definir seu ponto central. Uma das maneiras de executar essa ação seria usando a transformada de Hough para formas circulares, com o método HoughCircles (OpenCV) (Kapur and Thakkar, 2015). Porém, neste caso, as marcações fotogramétricas poderiam apresentar formas circulares imperfeitas, dependendo das condições de iluminação, posição e qualidade da câmera. Isto pode inviabilizar o uso da transformada de Hough. Contudo, existe uma abordagem alternativa para realizar esta operação utilizando um método de identificação de contornos, que foi empregado neste trabalho .

A detecção de contornos, portanto, é feita utilizando o método findContours (da biblioteca OpenCV), que retorna uma lista de matriz de pontos contendo todos os contornos encontrados na imagem. No método findContours é passado como parâmetro a flag $\boldsymbol{R} \boldsymbol{E} \boldsymbol{T R} \_\boldsymbol{L I S T}$, que limita a seleção apenas de contornos do primeiro nível de hierarquia, melhor dizendo, só contornos que não se encontram dentro de outros contornos identificados (Kapur and Thakkar, 2015).

\subsection{Identificação dos Pontos}

Para o cálculo dos ângulos necessários para a análise fotogramétrica, é preciso que o centro de cada marcação fotogramétrica seja identificado, pois é a partir das coordenadas centrais desses pontos que os segmentos de retas são definidos e, consequentemente, os ângulos são aferidos. A identificação dos pontos é realizada pelo método moments da biblioteca OpenCV, sendo que as informações de extremidade de cada contorno podem ser obtidas pela Equação 1:

$$
M_{p q}=\sum_{x, y} x^{p} y^{q} I(x, y)
$$

Em seguida, as coordenadas do centroide de cada contorno podem ser calculadas pela Equação 2:

$$
C=\frac{m_{10}}{m_{00}}, \frac{m_{01}}{m_{00}}
$$

Todos os pontos centrais das marcações fotogramétricas são então armazenados em um vetor de coordenadas de pontos.

\subsection{Cálculo dos Ângulos}

A aplicação visa fazer dois tipos de análise das medidas fotogramétricas relacionadas à escoliose: análise frontal na vista anterior e análise frontal na vista posterior. Neste caso, é necessário calcular os ângulos usando relações trigonométricas.

De acordo com o trabalho de Stolfi (2014), a verificação postural de presença de escoliose na coluna vertebral requer pontos de análise com duas referências anatômicas, na análise no plano frontal e na vista anterior. Estes pontos são a borda superior do Manúbrio e as espinhas ilíacas anteriores superiores que formam dois segmentos de reta oblíquos, os quais formam ângulos com a linha vertical como mostra a Figura 2.

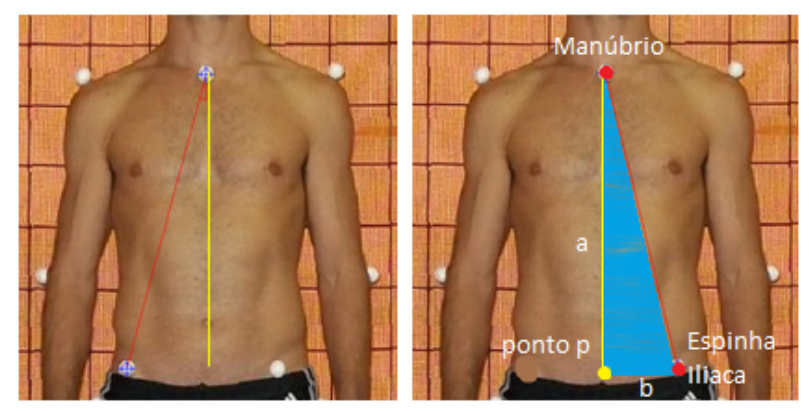

Figura 2. Segmentos de retas formados pelos pontos de análise caracterizados pelo Manúbrio e Espinhas Ilíacas.

Observando o contexto apresentado na avaliação frontal na vista anterior, identificou-se a possibilidade da formação de triângulos retângulos (Figura 2) a partir da definição de um terceiro ponto $P$, formado pela coordenada $Y$ do ponto referente à crista ilíaca e pela coordenada $X$ do ponto referente ao Manúbrio.

Isso permite calcular os ângulos formados pelo segmento de reta entre o Manúbrio e espinha Ilíaca a partir da relação trigonométrica dada pela distância entre a crista ilíaca e o ponto $P$, representado pela letra a, e a distância entre o Manúbrio e o ponto $P$, representada pela letra $\mathbf{b}$.

Dessa forma os dois ângulos desejados para a análise postural podem ser encontrados a partir da Equação 3:

$$
\alpha=\arctan \left(\frac{b}{a}\right)
$$

Para que fossem encontrados e demarcados os pontos fotogramétricos na análise frontal da vista posterior (Figura 3a), primeiramente foi demarcado o processo espinhoso de C7 (ponto P1 na Figura 3b). Posteriormente deve ser identificado o ponto inferior da escápula esquerda e direita e traçar uma linha imaginária unindo os dois pontos. Essa linha forma uma intersecção ao passar sobre a coluna vertebral, que deve ser marcada como um ponto de referência anatômica (ponto P2 na Figura 3b).

O mesmo procedimento feito para o ângulo inferior das escapulas deve ser feito para as espinhas ilíacas pósterosuperiores (EIPS). A partir da identificação das duas EIPS, traça-se a linha imaginária entre elas, demarcando a interseção da linha imaginária sobre a coluna vertebral (ponto P3 na Figura 3b). A análise da verticalização da coluna deve ser verificada a partir do ângulo formado por dois segmentos de retas formados pelos três pontos de referência anatômica: $\mathrm{C} 7$, interseção da linha formada pela escápula esquerda e direita com a coluna vertebral e a interseção formada pela linha imaginária entre as EIPS sobre a coluna vertebral como mostra a Figura 3a.

Avaliando o contexto apresentado na análise frontal na vista posterior, a solução proposta para a identificação dos ângulos necessários para a análise envolve a definição de mais três pontos na imagem, que formarão três novos tri- 
ângulos caracterizados como triângulos retângulos, como representado na figura $3 \mathrm{~b}$.

a]

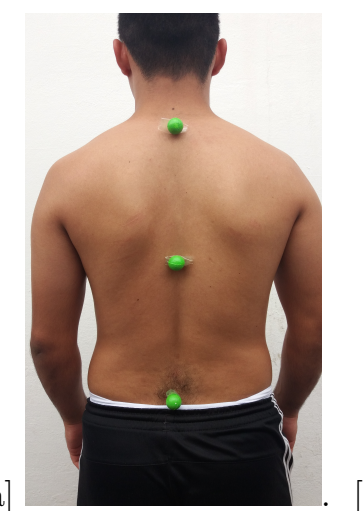

$[\mathrm{b}]$

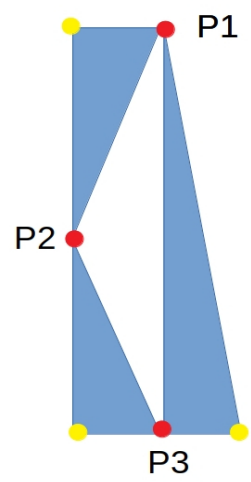

Figura 3. Referências anatômicas para análise postural da verticalização da coluna vertebral no plano frontal na vista posterior (a). Triângulos retângulos caracterizados com base em P1, P2 e P3 (b).

Na Figura 3b vemos os triângulos retângulos caracterizados a partir da definição de mais três pontos (representados pelos pontos amarelos) com base nos pontos de referência anatômica P1 (C7), P2 (interseção da linha formada pela escapula esquerda e direita com a coluna vertebral) e P3 (interseção formada pela linha imaginária entre as EIPS sobre a coluna vertebral) representados na cor vermelha.

Usando os três triângulos retângulos podemos aplicar o teorema de Pitágoras (Equação 4).

$$
a^{2}=b^{2}+c^{2}
$$

Com isso é possível encontrar a distância entre P1 e P2, entre P2 e P3 e entre P1 e P3. De posse desses valores, podemos então usar a lei dos cossenos (Equação 5) para encontrar os ângulos internos do triângulo formado por P1, P2 e P3.

$$
a^{2}=b^{2}+c^{2}-2 \cdot b \cdot c \cdot \cos (\alpha)
$$

Com o uso da lei dos cossenos, encontramos o ângulo formado pelos segmentos de retas que se encontram no ponto P2. Usando como base a linha vertical, considera-se como alinhamento ideal o ângulo de $180^{\circ}$.

\section{RESULTADOS}

Para validação da ferramenta construída nessa pesquisa, foram utilizadas imagens de procedimentos de avaliações posturais com fotogrametria colhidas na Internet. Infelizmente, não existe uma base de dados pública composta de material relativo a esse tipo de exame, portanto, ao todo foram encontradas apenas quatro imagens que cumpriram os critérios necessários para a realização da validação. Os critérios eram: possuir pontos fotogramétricos demarcados necessários para a avaliação na vista anterior ou na vista posterior; possuir os pontos fotogramétricos nítidos, sem manipulação ou alteração na imagem; e assegurar que os pontos foram demarcados por um profissional competente e habilitado.

Uma das imagens foi colhida diretamente do trabalho publicado de Stolfi (2014), e as outras três imagens foram obtidas na base de dados do site Treino em (escola.treinoemfoco.com.br), cujos autores das avaliações posturais foram os mesmos autores do trabalho descrito por Stolfi e Moura (2014).

O processo de validação consistiu na mensuração dos ângulos formado pelos segmentos de retas formados pelo Manúbrio e Espinhas Ilíacas, na análise frontal na vista anterior. Já na análise frontal na vista posterior, utilizouse como base os pontos de referência anatômica: C7 (P1 na Figura 3b); interseção da linha formada pela escápula esquerda e direita com a coluna vertebral (P2 na Figura 3b); e interseção formada pela linha imaginária entre as EIPS sobre a coluna vertebral (P3 na Figura 3b). A avaliação das quatro imagens obtidas foi realizada no software High Posture através da ferramenta de ângulos livres e também na aplicação proposta por essa pesquisa. O software High Posture foi escolhido para comparação por também usar como base medidas fotogramétricas e por se caracterizar como o software mais citado em estudos relacionados. Ambas avaliações foram conduzidas por um profissional formado em fisioterapia.

A Tabela 1 exibe as avaliações posturais de quatro indivíduos, sendo uma das imagens da vista anterior, do indivíduo 1 e as demais na vista posterior. Na Coluna SAPO, são exibidos os resultados obtidos através da análise das imagens do software $S A P O$, fazendo uso da ferramenta de ângulos livres. Na Coluna High Posture são apresentados os resultados obtidos pelo aplicativo que representa a abordagem proposta descrita na sessão 2. Sendo High Posture o nome adotado para a ferramenta desenvolvida nesse trablho.

Tabela 1. Resultados obtidos pelo software SAPO e pela ferramentaHigh Posture (Método proposto pelo presente trabalho).

\begin{tabular}{c|c|c|c} 
Indivíduo & SAPO & High Posture* & Tipo de Avaliação \\
\hline 1 & $1,4^{\circ}$ & $1,72^{\circ}$ & Vista Anterior \\
\hline 2 & $173,1^{\circ}$ & $173,19^{\circ}$ & Vista Posterior \\
\hline 3 & $172,1^{\circ}$ & $173,62^{\circ}$ & Vista Posterior \\
\hline 4 & $171,5^{\circ}$ & $170,59^{\circ}$ & Vista Posterior
\end{tabular}

Podemos observar que, na análise do indivíduo 1 (vista anterior), a avaliação obtida pelo software $S A P O$ informou um desbalanceamento de $1,4^{\circ}$, enquanto que a avaliação da aplicação High Posture, obteve como resultado $1,72^{\circ}$. Ou seja, houve discordância de aproximadamente $0,3^{\circ}$.

$\mathrm{Na}$ análise dos indivíduos 2,3 e 4 (vista posterior), a avaliação obtida pelo software $S A P O$ resultou em ângulos de $173.1^{\circ}, 172.1^{\circ}, 171.5^{\circ}$, respectivamente. Já a avaliação da aplicação High Posture, obteve os respectivos resultados: $173.19^{\circ}, 173.62^{\circ} \mathrm{e} 170.59^{\circ}$. Caracterizando diferença de aproximadamente $0,09^{\circ}, 1.52^{\circ}$ e $0.91^{\circ}$ nos resultados obtidos pelas duas aplicações.

\section{DISCUSSÃO}

Considerando os dados obtidos na primeira tentativa de validação da ferramenta, observou-se uma média de variação entre os resultados obtidos próxima de $0.84^{\circ}$. O que sugere um bom desempenho da ferramenta, tendo como parâmetro os resultados de Stolfi (2014), que encontraram coeficientes de variação próximos a 1,6\% inter- 
examinadores, na aferição da verticalização da coluna vertebral, tendo como referência $180^{\circ}$.

Um aspecto de impacto no resultado é que as imagens utilizadas não apresentavam qualidade expressiva. Esse fator é determinante para a qualidade da detecção na abordagem proposta pelo presente trabalho(High Posture). Entretanto, a aplicação apresentou bons resultados apesar da baixa qualidade das imagens, logo, espera-se que o seu desempenho seja mais preciso com imagens captadas diretamente dos sensores.

No processo de desenvolvimento desse trabalho, foram constatadas algumas dificuldades nas abordagens propostas para a solução do problema apresentado. Em primeiro momento, o método da transformada de Hough que foi utilizado inicialmente na detecção de formas circulares não apresentou resultados aceitáveis quando submetido a imagens usadas para teste. Por esse motivos, optamos pelo método de segmentação por cores.

Outra dificuldade está relacionada ao método de segmentação baseado no nível de luminosidade. Nesse caso alguns fatores como o nível de luz no ambiente e a tonalidade da pele do indivíduo avaliado podem fazer com que áreas da imagem que não foram marcadas como referência anatômica sejam detectadas como tais, inviabilizando a avaliação dos ângulos necessários para a aferição do alinhamento postural. Dessa forma, para o funcionamento da aplicação, definimos que as marcações seriam da cor verde, para haver maior possibilidade de contraste com a pele dos indivíduos.

\section{CONCLUSÃO}

Ao final da pesquisa, o desenvolvimento do algoritmo listado como primeiro objetivo específico foi alcançado seguindo a metodologia descrita na seção 2 . O segundo objetivo específico, que consiste na construção da aplicação móvel também foi alcançado. O terceiro objetivo específico foi alcançado parcialmente, pois a validação do aplicativo construído não ocorreu com a participação de voluntários. Foi realizada uma solicitação de autorização ao conselho de ética local, contudo, a entidade exigiu que fossem realizadas adequações ao trabalho. Após realizadas as alterações requisitadas, o processo foi reenviado para análise do conselho, que não divulgou resposta até o presente momento. Esta situação impossibilitou, temporariamente, a validação por meio de uma avaliação postural real, portanto, a validação foi realizada a partir de imagens colhidas em artigos e sítios especializados em avaliação postural.

Considerando a questão de pesquisa apresentada, os resultados obtidos na validação do aplicativo reforçam a ideia de que o processamento de imagem em smartphones é capaz de identificar de forma eficaz medidas fotogramétricas correspondentes aos pontos anatômicos, possibilitando a identificação e acompanhamento do desvio postural caracterizado como escoliose. Considerando, obviamente, que a ferramenta desenvolvida deva ser utilizada por um profissional da saúde especializado em avaliação postural.

O aplicativo desenvolvido nessa pesquisa é capaz de identificar duas medidas de ângulos referentes a vista anterior do tronco, e uma medida de ângulo referente a vista posterior. Embora essas medidas sejam muito relevantes, a identificação e acompanhamento da escoliose de forma completa envolve o aferimento de um número maior de pontos anatômicos.

\section{REFERÊNCIAS}

Duarte M., Ferreira E.A., M.E.F.A. (2005). Documentação sobre o SAPO -Software para avaliação postural. URL http: //demotu .org/sapo2/SAPOdoc.pdf.

Hopkins, B.B., Vehrs, P.R., Fellingham, G.W., George, J.D., Hager, R., and Ridge, S.T. (2019). Validity and reliability of standing posture measurements using a mobile application. Journal of manipulative and physiological therapeutics.

Iacob, S.M., Chisnoiu, A.M., Lascu, L.M., Berar, A.M., Studnicska, D., and Fluerasu, M.I. (2018). Is posturescreen $\AA$ mobile app an accurate tool for dentists to evaluate the correlation between malocclusion and posture? CRANIOR, 1-7.

Kapur, S. and Thakkar, N. (2015). Mastering OpenCV Android Application Programming. Packt Publishing Ltd.

Mazzuia, A.R.d.O., Machado, D.R., Fukumothi, D.K., Nunes, L.F.B., Tucci Neto, C., Jorge, H.M.d.H., Ortiz, R.T., and Mattos, C.A.d. (2015). Iphone app use to cobb angle in adolescent idiopathic scoliosis: Does this apply? Coluna/Columna, 14(2), 101-104.

Nery, P.B. (2009). Análise da confiabilidade intra e interexaminador do software de avaliação postural-SAPO em escolares do município de Ribeirão Preto-SP. Ph.D. thesis, Universidade de São Paulo.

Shaw, M., Adam, C.J., Izatt, M.T., Licina, P., and Askin, G.N. (2012). Use of the iphone for cobb angle measurement in scoliosis. European Spine Journal, 21(6), 1062-1068.

Souza, J., Pasinato, F., Basso, D., Corrêa, E., and da Silva, A.M. (2011). Biophotogrammetry: reliability of measurements obtained with a posture assessment software (sapo). Brazilian Journal of Kinanthropometry and Human Performance, 13(4), 299-305. doi:10.5007/ 1980-0037.2011v13n4p299.

Srinivasalu, S., Modi, H.N., SMehta, S., Suh, S.W., Chen, T., and Murun, T. (2008). Cobb angle measurement of scoliosis using computer measurement of digitally acquired radiographs-intraobserver and interobserver variability. Asian spine journal, 2(2), 90-93.

Stolfi, Jéssica, M.J.A.d. (2014). Confiabilidade intra e interexaminadores de medidas fotogramétricas para análise do alinhamento vertical da coluna vertebral. Revista de Atenção à Saúde (antiga Rev. Bras. Ciên. Saúde), 12(41).

Suken A. Shah, M. (2009). What is scoliosis? URL https://www.nemours.org/content/dam/nemours/ www/filebox/service/medical/orthopedics/ scoliosis.pdf.

Szucs, K.A. and Brown, E.V.D. (2018). Rater reliability and construct validity of a mobile application for posture analysis. Journal of physical therapy science, 30(1), 3136 . 